\title{
Superlinear elliptic problems with sign changing coefficients
}

\author{
Eugenio Massa $^{a, b, *}$ and Pedro Ubilla ${ }^{b, \dagger}$ \\ ${ }^{a}$ Departamento de Matemática, Instituto de Ciências Matemáticas e de Computação, \\ Universidade de São Paulo - Campus de São Carlos, Caixa Postal 668, 13560-970, São Carlos SP, Brazil. \\ ${ }^{b}$ Departamento de Matemáticas y C. C., Universidad de Santiago de Chile, \\ Casilla 307, Correo 2, Santiago, Chile.
}

Corresponding author: Pedro Ubilla, e-mail: pubilla@fermat.usach.cl

address for manuscript correspondence: Departamento de Matemáticas y C. C., Universidad de Santiago de Chile,Casilla 307, Correo 2, Santiago, Chile.

Tel +562 7182069, Fax +5626813125.

*The author was partially supported by Fapesp / Brazil.

${ }^{\dagger}$ The author was partially supported by FONDECYT N ${ }^{0} 1080430$.

${ }^{0}$ e-mail addresses: eug.massa@gmail.com (E. Massa) pubilla@fermat.usach.cl (P. Ubilla) 


\begin{abstract}
Via variational methods, we study multiplicity of solutions for the problem

$$
\begin{cases}-\Delta u=\lambda b(x)|u|^{q-2} u+a u+g(x, u) & \text { in } \Omega, \\ u=0 & \text { on } \quad \partial \Omega .\end{cases}
$$

where a simple example for $g(x, u)$ is $|u|^{p-2} u$; here $a, \lambda$ are real parameters, $1<q<2<$ $p \leq 2^{*}$ and $b(x)$ is a function in a suitable space $L^{\sigma}$. We obtain a class of sign changing coefficients $b(x)$ for which two non-negative solutions exist for any $\lambda>0$, and a total of five nontrivial solutions are obtained when $\lambda$ is small and $a \geq \lambda_{1}$. Note that this type of results are valid even in the critical case.
\end{abstract}

Keywords and phrases: multiplicity of solutions, variational methods, subcritical and critical growth, concave-convex nonlinearity, sign changing coefficients.

\title{
1 Introduction
}

We study a class of problems of which a simple but significative example is the following:

$$
\begin{cases}-\Delta u=\lambda b(x)|u|^{q-2} u+a u+|u|^{p-2} u & \text { in } \Omega \\ u=0 & \text { on } \quad \partial \Omega\end{cases}
$$

where $\Omega \subset \mathbb{R}^{N}$ is an open bounded domain with smooth boundary $\partial \Omega, a \in \mathbb{R}, \lambda>0$ are real parameters, and the function $b(x)$ may change sign and be unbounded.

When $b(x) \equiv 1$ and $a=0$, one has the classical "concave-convex" nonlinearity considered in [1]. They proved the existence of a value $\Lambda>0$ such that there exist at least two positive solutions for $0<\lambda<\Lambda$, at least one for $\lambda=\Lambda$ and no positive solution for $\lambda>\Lambda$. In this case, one of the solutions was obtained, without growth restrictions, using sub and supersolutions; the other one was obtained in the subcritical and the critical case, via the Mountain Pass Theorem.

On the other hand, if $b(x) \equiv-1$ and the superlinear term is subcritical, one non-negative solution for any $a \in \mathbb{R}$ and $\lambda>0$ was found in [2], via Mountain Pass Theorem.

If $b(x)$ is a function in a suitable space $L^{\sigma}$ (possibly sign changing) and the superlinear term is subcritical, the results in [3] imply that there still exist two non-negative solutions for small $\lambda$; in this case the solutions are obtained via Mountain Pass and minimization in a small ball.

In the case of a critical superlinearity, that is, when $p=2^{*}=\frac{2 N}{N-2}$, the result in [1] still holds, while the case of a variable coefficient $b(x)$ was considered in [4], finding again two positive solutions but under the condition $b \in L^{\infty}$ and $b \geq 0$; the latter requirement is important because it implies a monotone dependence on $\lambda$ of the nonlinearity, which is crucial for the application of the argument of sub and supersolutions.

If one considers $a \geq \lambda_{1}$ (the first eigenvalue of the Laplacian in $\Omega$ ) and a more general superlinear term in place of $|u|^{p-2} u$, then it becomes interesting to look also for sign changing solutions. In fact, observe that if we set $a>\lambda_{1}$ in Problem (1.1), then a non-negative solution may exist only if $b(x)$ is negative at least in a region of positive measure.

When $b(x) \equiv-1$, in the already cited [2], it was proved that, along with a non-negative (and a non-positive) nontrivial solution, a further solution exists provided $\lambda$ is small enough and $a \geq \lambda_{1}$; this third solution is obtained via Linking Theorem. 
On the other hand, using Morse theory, two nontrivial solutions are obtained in [5] for $b(x) \equiv 1$, and one nontrivial solution is obtained in [6] with a sign changing coefficient $b \in L^{\infty}$, provided $a$ is below a certain value depending on $b(x)$.

A consequence of the main result in this paper is the existence, in the subcritical and critical case, of five nontrivial solutions of Problem (1.1), of which two are non-negative and two nonpositive, for a suitable sign changing coefficient $b(x)$.

In the following, we consider a larger class of problems with respect to (1.1):

$$
\begin{cases}-\Delta u=\lambda f_{\mu}(x, u)+a u+g(x, u) & \text { in } \Omega \\ u=0 & \text { on } \partial \Omega\end{cases}
$$

where now $g$ is a superlinear term (possibly critical), $\mu \geq 0$ a further parameter and $f_{\mu}(x, u)$ a suitable sublinear term, which may change sign.

Our first purpose is to determine a class of functions $f_{\mu}(x, u)$, for which the results in [2] are still valid, that is, for which we may obtain at least a non-negative solution and a non-positive one for any $\lambda>0$ and $a \in \mathbb{R}$, and a third solution for $\lambda$ small and $a \geq \lambda_{1}$; as observed above, in order to obtain this, one needs $f_{\mu}(x, s) s<0$ in some region. However, it turns out that if $f_{\mu}(x, s) s>0$ in some other region, which was not the case in [2], then one more non-positive (and one non-negative) solution arises. This will be shown in Theorems 2.1 and 2.2.

Our second purpose is to study this problem in the more delicate critical case, which was not considered in [2]: this result will be given in Theorems 2.4 and 2.5. Observe also that in [3] were considered, like here, unbounded coefficients, however, the critical case was not studied, and so our work complements, in some sense, also that result.

As is well known, when the nonlinearity has this critical behavior, the functional associated to the equation does not, in general, satisfy the Palais-Smale (PS) condition; therefore, it is necessary to first show that this condition is still satisfied at suitable levels, and then to prove that the critical levels coming from the variational characterization in the Mountain Pass or Linking Theorem actually lie at these levels. For these purposes we will use some techniques derived from $[7,1,8]$. See also in [9].

Other problems related to our (1.1) and (1.2) have been considered in several other works. In [10], Perera considers the case $a>\lambda_{1}$ when the coefficient $b \equiv-1$, but with different hypotheses on the behavior of the nonlinearity at infinity which impose a interaction with the first eigenvalue; in this case, a key role is played by the coercivity of the functional and he obtains up to five nontrivial solutions (of which two non positive and two non-negative). In [11] and [12], the coefficient $b$ is assumed in $L^{\infty}$ but is allowed to change sign and the nonlinearity is asymptotically linear: in [11] a nontrivial solution is obtained for $a$ slightly above $\lambda_{1}$, while in [12] the case $a<\lambda_{1}$ is considered and two non-negative solutions are obtained.

The paper is organized as follows: in Section 2, we give the precise statement of the results, in the Sections 3 and 4 we give the proofs of the main theorems: Section 3 is devoted to the subcritical case and Section 4 to the critical case; finally, in Section 5 , we consider the case of a different set of hypotheses on the function $f_{\mu}$. 


\section{Results}

We will consider two cases for the term $f_{\mu}(x, u)$ : the first one is

$$
f_{\mu}(x, u)=b_{\mu}(x)|u|^{q-2} u \text { with } 1<q<2,
$$

where we write $b_{\mu}(x)=c_{\mu}(x)-d(x)$, with $c_{\mu}, d \geq 0$.

In the second one, we will consider similar coefficients $\bar{c}_{\mu}, \bar{d} \geq 0$, but multiplying terms with different exponents, namely

$$
f_{\mu}(x, u)=\bar{c}_{\mu}(x)|u|^{q_{1}-2} u-\bar{d}(x)|u|^{q_{2}-2} u \text { with } 1<q_{1}<q_{2}<2 .
$$

For the case $\left(f_{1}\right)$, we will assume the following set of hypotheses:

$\left(H f_{a}\right) c_{\mu}, d \in L^{\sigma}(\Omega)$ for some $\sigma>\frac{2^{*}}{2^{*}-q}$, for every $\mu \geq 0$;

$\left(H f_{b}\right)$ there exists $\delta_{d}>0$ such that $d(x) \geq \delta_{d}>0$ in $\Omega$;

$\left(H f_{c}\right)\left\|c_{\mu}\right\|_{L^{\sigma}}$ is a continuous function of $\mu \in[0,+\infty)$ and $\lim _{\mu \rightarrow 0}\left\|c_{\mu}\right\|_{L^{\sigma}}=0$;

$\left(H f_{d}\right)$ for every $\mu>0$, there exist an open set $\Omega_{\mu} \subseteq \Omega$ and a constant $M_{\mu}>0$ such that

$$
c_{\mu}(x) \geq d(x)+M_{\mu} \text { in } \Omega_{\mu} .
$$

A model for this coefficient could be $b_{\mu}(x)=(\mu c(x)-1)$, where $c \in L^{\sigma}(\Omega), c \geq 0$, and there exists $x_{0} \in \Omega$ such that $c$ is continuous in $\bar{\Omega} \backslash\left\{x_{0}\right\}$ and $\lim _{x \rightarrow x_{0}} c(x)=+\infty$; a simple example is $\frac{1}{\left|x-x_{0}\right|^{\gamma}}$ with $\gamma<\frac{N\left(2^{*}-q\right)}{2^{*}}$. Note that condition $\left(H f_{c}\right)$ with $\mu=0$ implies $c_{\mu} \equiv 0$.

Observe that the given hypotheses imply that, when the parameter $\mu$ goes to zero, the region where $b_{\mu}>0$ reduces to zero in measure, but never vanishes. However, this set needs not reduce to a single small ball: for instance, we could also choose, in the above expression for $b_{\mu}$, the function $c(x)=\sum_{j=1}^{\infty} 2^{-j} \frac{1}{\left|x-x_{j}\right| \gamma}$ with $\gamma$ as above and $\left\{x_{j}: j \in \mathbb{N}\right\}$ a dense subset of $\Omega$; in this case, the coefficient $b_{\mu}(x)$ is positive in a subset of any open set, for any $\mu>0$.

For the case $\left(f_{2}\right)$, we will assume

$\left(H f_{a}^{\prime}\right) \bar{c}_{\mu} \in L^{\sigma_{1}}(\Omega)$ and $\bar{d} \in L^{\sigma_{2}}(\Omega)$ for values $\sigma_{i}>\frac{2^{*}}{2^{*}-q_{i}}(i=1,2)$, for every $\mu \geq 0$;

$\left(H f_{d}^{\prime}\right)$ for every $\mu>0$, there exist an open set $\Omega_{\mu} \subseteq \Omega$ and a constant $M_{\mu}>0$ such that

$$
\bar{c}_{\mu}(x) \geq M_{\mu} \text { and } \bar{d}(x) \text { is bounded in } \Omega_{\mu} ;
$$

and $\bar{c}_{\mu}, \bar{d}$ satisfy $\left(H f_{b}\right)$ and $\left(H f_{c}\right)$ (with the $L^{\sigma_{1}}$ norm instead of $\left.L^{\sigma}\right)$.

Observe that the major difference between the two cases, lies in assumption $\left(H f_{d}\right)$, actually, in case $\left(f_{1}\right)$ this assumption implies, because of $\left(H f_{b}\right)$, that $c_{\mu} \notin L^{\infty}$; this is not required in case $\left(f_{2}\right)$ due to the assumption $q_{1}<q_{2}$, so in this case we just need to ask, in $\left(H f_{d}^{\prime}\right)$, that, in some region, $\bar{c}_{\mu}$ does not vanish and $\bar{d}$ is bounded.

Throughout the paper, we will denote by $0<\lambda_{1}<\lambda_{2} \leq \lambda_{3} \leq \ldots \leq \lambda_{k} \leq \ldots$ the eigenvalues of $-\Delta$ in $H_{0}^{1}(\Omega)$, by $\sigma(-\Delta)=\left\{\lambda_{k}: k \in \mathbb{N}\right\}$ its spectrum and by $\left\{\phi_{k}\right\}_{k \in \mathbb{N}}$ the corresponding eigenfunctions, taken orthogonal and normalized with $\left\|\phi_{k}\right\|_{L^{2}}=1$ and $\phi_{1}>0$.

It is worth pointing out that for Problem (1.2), nontrivial solutions may vanish on a nonempty open set (see for example in [13]), due to the term $f_{\mu}(x, u)$, which may violate the hypotheses of the Hopf's Lemma if in some region $\lim _{s \rightarrow 0} \frac{f_{\mu}(x, s)}{s}=-\infty$ (that is, in case $\left(f_{1}\right)$, if $b_{\mu}<0$ ); for this reason our results will concern non-negative and non-positive solutions, rather than strictly positive or negative ones. 


\subsection{Results for the subcritical case}

For the subcritical case, as anticipated above, we will consider in Problem (1.2) a superlinear term in the more general form $g(x, s)$, satisfying the following hypotheses:

$\left(H g_{0}\right) g: \Omega \times \mathbb{R} \rightarrow \mathbb{R}$ is a Carathéodory function;

$\left(H g_{1}\right)$ there exists $p \in\left(2,2^{*}\right)$ and $C \in \mathbb{R}$ such that $|g(x, s)| \leq C\left(1+|s|^{p-1}\right)$;

$\left(H g_{2}\right)(i) \lim _{s \rightarrow \pm \infty} \frac{g(x, s)}{s}=+\infty$ uniformly;

(ii) there exists $\Theta>2$ and $s_{0} \geq 0$ such that $\Theta G(x, s)-s g(x, s)+\left(\frac{\Theta}{2}-1\right) a s^{2} \leq 0$ for $|s| \geq s_{0}$;

$\left(H g_{3}\right) \lim _{s \rightarrow 0} \frac{g(x, s)}{s}=0$ uniformly.

Our first results are

Theorem 2.1. Let $f_{\mu}$ satisfy $\left(H f_{a} \ldots H f_{d}\right)$ (or the corresponding ones for case $\left.\left(f_{2}\right)\right), g$ satisfy $\left(H g_{0} \ldots H_{3}\right)$ and $a \in \mathbb{R}$. Then, for every $\lambda>0$ there exists $\bar{\mu}(\lambda)$ such that Problem (1.2) has two nontrivial non-negative solutions and two nontrivial non-positive solutions for $\mu \in(0, \bar{\mu}(\lambda))$.

Theorem 2.2. Let $f_{\mu}$ satisfy $\left(H f_{a} \ldots H f_{d}\right)$ (or the corresponding ones for case $\left(f_{2}\right)$ ), g satisfy $\left(\begin{array}{lll}H g_{0} & \ldots & H g_{3}\end{array}\right)$ and moreover $G(x, u)=\int_{0}^{u} g(x, s) d s \geq 0$ and $a \geq \lambda_{1}$. Let $A>0$ be arbitrary. Then there exists $\bar{\lambda}(A)>0$ such that Problem (1.2) has a further nontrivial solution for $\lambda \in[0, \bar{\lambda}(A))$ and $\mu \in[0, A)$.

In particular, for $\lambda \in(0, \bar{\lambda}(A))$ and $\mu \in(0, \min \{\bar{\mu}(\lambda), A\})$, we have at least 5 nontrivial solutions.

Remark 2.3. Observe that the role of the two parameters is quite different in the two results: the four solutions in Theorem 2.1 exist for any $\lambda>0$ and small $\mu>0$, while the further solution in Theorem 2.2 exists for any $\mu>0$ and small $\lambda>0$. However, since $\bar{\lambda}(A)$ does not depend directly on $\mu$, but just on the bounded set where we limit $\mu$, one obtains a region in the quadrant $\{\mu, \lambda>0\}$ where all the five nontrivial solutions exist.

\subsection{Results for the critical case}

In the critical case, we will need to consider a simpler form for the superlinear term, in fact, we will take $g(x, u)=|u|^{p-2} u$ where $p=2^{*}=\frac{2 N}{N-2}$; so the problem reads

$$
\begin{cases}-\Delta u=\lambda f_{\mu}(x, u)+a u+|u|^{p-2} u & \text { in } \quad \Omega, \\ u=0 & \text { on } \partial \Omega .\end{cases}
$$

Our results in the critical case are

Theorem 2.4. Let $N \geq 3, f_{\mu}$ satisfy $\left(H f_{a} \ldots H f_{d}\right)$ (or the corresponding ones for case $\left(f_{2}\right)$ ), $p=2^{*}$ and $a>0$. Then, for every $\lambda>0$ there exists $\bar{\mu}(\lambda)$ such that Problem (2.1) has two nontrivial non-negative solutions and two nontrivial non-positive solutions for $\mu \in(0, \bar{\mu}(\lambda))$. 
Theorem 2.5. Let $N \geq 4, f_{\mu}$ satisfy $\left(H f_{a} \ldots H f_{d}\right)$ (or the corresponding ones for case $\left(f_{2}\right)$ ), $p=2^{*}$ and moreover $a>\lambda_{1}$ and $a \notin \sigma(-\Delta)$. Let $A>0$ be arbitrary. Then there exists $\bar{\lambda}(A)>0$ such that Problem (2.1) has two further nontrivial solutions for $\lambda \in[0, \bar{\lambda}(A))$ and $\mu \in[0, A)$. In particular, for $\lambda \in(0, \bar{\lambda}(A))$ and $\mu \in(0, \min \{\bar{\mu}(\lambda), A\})$, we have at least six nontrivial solutions.

As stated in the introduction, in [2] the critical case was not considered, so that we can complement that result by considering the case $f_{\mu}(x, u)=-|u|^{q-2} u$, which corresponds to our Problem (2.1) with $\mu=0$ and $d(x) \equiv-1$; in fact we have

Theorem 2.6. Under the same hypotheses of Theorem 2.5, Problem (2.1) has one non-negative nontrivial solution, one non-positive, and two further nontrivial solutions, for $\mu=0$ and $\lambda>0$ small enough.

Remark 2.7. We observe that the result for the case of Theorem 2.6 is not as complete as it is when $\mu \neq 0$, actually, the non-negative solution is guaranteed only for small $\lambda$, and not for any $\lambda>0$ as in Theorem 2.4. In fact, in the proof of Theorem 2.4, the existence of a region where $b_{\mu}>0$ allows us to obtain, for any $\lambda>0$, a mountain pass level where the (PS) condition holds, something that we cannot do with the strictly negative coefficient.

\section{The subcritical case}

We will consider the following $\mathcal{C}^{1}$-functionals defined in $H_{0}^{1}(\Omega)$ :

$$
J_{\mu, \lambda}(u)=\frac{1}{2}\left(\|u\|^{2}-a\|u\|_{2}^{2}\right)-\int_{\Omega} \lambda F_{\mu}(x, u)-\int_{\Omega} G(x, u)
$$

and

$$
J_{\mu, \lambda}^{ \pm}(u)=\frac{1}{2}\left(\|u\|^{2}-a\left\|u^{ \pm}\right\|_{2}^{2}\right)-\int_{\Omega} \lambda F_{\mu}\left(x, u^{ \pm}\right)-\int_{\Omega} G\left(x, u^{ \pm}\right),
$$

where $\|\cdot\|$ represents the norm in $H_{0}^{1},\|\cdot\|_{r}$ the norm in $L^{r}, F_{\mu}(x, u)=\int_{0}^{u} f_{\mu}(x, s) d s$ and $G(x, u)=$ $\int_{0}^{u} g(x, s) d s$.

Critical points of $J_{\mu, \lambda}^{+}$are non-negative solutions, while critical points of $J_{\mu, \lambda}^{-}$are non-positive solutions; then they are also critical points of $J_{\mu, \lambda}$ at the same level.

Throughout the proofs, $C, C^{\prime}$ will be used to denote several constants whose exact value is irrelevant. Also, we will consider just the case $\left(f_{1}\right)$ during the proofs, and discuss the case $\left(f_{2}\right)$ at the end of the paper, in section 5.

First of all, we prove some estimates which will be used throughout the following proofs.

\section{Lemma 3.1.}

(a) If $\left(H g_{1}\right)$ and $\left(H g_{3}\right)$ hold, then for any $\varepsilon>0$ there exists $D_{\varepsilon}$ such that

$$
|G(x, s)| \leq \varepsilon \frac{s^{2}}{2}+D_{\varepsilon} \frac{|s|^{p}}{p}, \quad \text { for all } s \in \mathbb{R} .
$$

(b) If $\left(H g_{1}\right)$ and $\left(H g_{2}\right)-(i)$ hold, then for any $M>0$ there exists $E_{M}$ such that

$$
G(x, s) \geq M \frac{s^{2}}{2}-E_{M}, \quad \text { for all } s \in \mathbb{R} .
$$


Proof. The hypothesis $\left(H g_{3}\right)$ implies that there exists $s_{1}(\varepsilon)$ such that $|G(x, s)| \leq \varepsilon \frac{s^{2}}{2}$ for $|s| \leq$ $s_{1}(\varepsilon)$, while $\left(H g_{1}\right)$ implies that $|G(x, s)| \leq C|s|+C \frac{|s|^{p}}{p}$ for any $s \in \mathbb{R}$, and so there exists $D_{\varepsilon}$ such that $|G(x, s)| \leq D_{\varepsilon} \frac{|s|^{p}}{p}$ for $|s| \geq s_{1}(\varepsilon)$. Then $|G(x, s)|$ is bounded by the sum of the two estimates for any $s \in \mathbb{R}$.

On the other hand, the hypothesis $\left(H g_{2}\right)-(i)$ implies that there exists $s_{2}(M)$ such that $G(x, s) \geq M \frac{s^{2}}{2}$ for $|s| \geq s_{2}(M)$, while $\left(H g_{1}\right)$ implies that there exists $E_{M}$ such that $G(x, u)-$ $M \frac{s^{2}}{2} \geq-E_{M}$ for $|s| \leq s_{2}(M)$. Then $G(x, u)-M \frac{s^{2}}{2} \geq-E_{M}$ for any $s \in \mathbb{R}$.

Now, we will study the (PS) condition for the functionals (3.1) and (3.2). We recall that a $\mathcal{C}^{1}$-functional $J$ is said to satisfy the $(\mathrm{PS})_{c}$ condition if any sequence in $H_{0}^{1}(\Omega)$ satisfying $J\left(u_{n}\right) \rightarrow c$ and $J^{\prime}\left(u_{n}\right) \rightarrow 0$ contains a convergent subsequence; it is said to satisfy the (PS) condition if $(\mathrm{PS})_{c}$ is satisfied for any $c \in \mathbb{R}$. We will prove

Lemma 3.2. Under hypotheses $\left(H g_{0} \ldots H_{2}\right)$ and $\left(H f_{a}\right)$, the three functionals in (3.1) and (3.2) satisfy the (PS) condition for any $\lambda, \mu \geq 0$ and $a \in \mathbb{R}$.

Proof. Let $\Theta>2$ be as in $\left(H g_{2}\right)-(i i)$; observe that by $\left(H g_{1}\right)$ we may reformulate $\left(H g_{2}\right)-(i i)$ as

$$
\Theta G(x, s)-s g(x, s)+\left(\frac{\Theta}{2}-1\right) a s^{2} \leq C \text { for any } s \in \mathbb{R} .
$$

Suppose $u_{n}$ is a (PS) sequence for $J_{\mu, \lambda}$, that is, $\left|J_{\mu, \lambda}\left(u_{n}\right)\right|<C$ and $\left|J_{\mu, \lambda}^{\prime}\left(u_{n}\right)\right| \leq \varepsilon_{n}\left\|u_{n}\right\|$ for some sequence $\varepsilon_{n} \rightarrow 0$; in order to prove that $u_{n}$ is bounded we estimate

$$
\begin{aligned}
\Theta C+\varepsilon_{n}\left\|u_{n}\right\| \geq \Theta J_{\mu, \lambda}\left(u_{n}\right) & -J_{\mu, \lambda}^{\prime}\left(u_{n}\right)\left[u_{n}\right]=\left(\frac{\Theta}{2}-1\right)\left(\left\|u_{n}\right\|^{2}-a\left\|u_{n}\right\|_{2}^{2}\right)+ \\
& -\int_{\Omega}\left(\Theta G\left(x, u_{n}\right)-g\left(x, u_{n}\right) u_{n}\right)-\lambda\left(\frac{\Theta}{q}-1\right) \int_{\Omega} b_{\mu}(x)\left|u_{n}\right|^{q}
\end{aligned}
$$

using (3.5) we get

$$
\left(\frac{\Theta}{2}-1\right)\left\|u_{n}\right\|^{2} \leq C^{\prime}+\varepsilon_{n}\left\|u_{n}\right\|+\lambda\left(\frac{\Theta}{q}-1\right)\left\|b_{\mu}\right\|_{\sigma}\left\|u_{n}\right\|^{q}
$$

and then $\left\|u_{n}\right\|$ is bounded.

The existence of a convergent subsequence now follows by classical arguments, since $p<2^{*}$ in $\left(H g_{1}\right)$ and $\sigma>\frac{2^{*}}{2^{*}-q}$ in $\left(H f_{a}\right)$.

The same argument works for $J_{\mu, \lambda}^{ \pm}$.

In the following two lemmas we will provide the geometry which allows to prove Theorem 2.1; first, in Lemma 3.3 we will find a "range of mountain" around the origin, and then, in Lemma 3.4, we will see that the origin cannot be a minimum.

Lemma 3.3. Under the hypotheses of Theorem 2.1, for every $\lambda>0$, there exists $\bar{\mu}(\lambda)$ such that for $\mu \in[0, \bar{\mu}(\lambda))$ there exist $r, \delta>0$ (depending on $\mu, \lambda$ ) such that $J_{\mu, \lambda}(u) \geq \delta>0$ for $\|u\|=r$. Moreover, $J_{\mu, \lambda}$ is bounded from below in the ball $B_{r}=\left\{u \in H_{0}^{1}:\|u\| \leq r\right\}$.

The same property holds for $J_{\mu, \lambda}^{ \pm}(u)$. 
Proof. In this proof we will separate the functional into two parts, where the dominant positive terms will be, respectively, $\lambda \int_{\Omega} d(x)|u|^{q}$ and $\|u\|^{2}$.

If $u \in \mathcal{C}_{0}^{1}(\Omega)$, using $\left(H f_{b}\right)$ and estimate (3.3) we get

$$
J_{1}(u):=\frac{\lambda}{q} \int_{\Omega} d(x)|u|^{q}-\frac{a}{2} \int_{\Omega} u^{2}-\int_{\Omega} G(x, u) \geq\|u\|_{q}^{q}\left(\frac{\lambda \delta_{d}}{q}-\frac{(a+\varepsilon)\|u\|_{\infty}^{2-q}}{2}-\frac{D_{\varepsilon}\|u\|_{\infty}^{p-q}}{p}\right) .
$$

For small $\|u\|_{\infty}$ the term in parentheses is positive, then 0 is a local minimum in the $\mathcal{C}^{1}$ topology and then also in the $H_{0}^{1}$ topology, by [14], that is, there exists $\rho(\lambda)>0$ such that $J_{1}(u) \geq 0$ for $\|u\| \leq \rho(\lambda)$.

For the remaining part of $J_{\mu, \lambda}$ we estimate

$$
J_{2}(u):=\frac{1}{2}\|u\|^{2}-\frac{\lambda}{q} \int_{\Omega} c_{\mu}(x)|u|^{q} \geq \frac{1}{2}\|u\|^{2}-\frac{\lambda\left\|c_{\mu}\right\|_{\sigma}}{q}\|u\|^{q} \geq\|u\|^{q}\left(\frac{\|u\|^{2-q}}{2}-\frac{\lambda\left\|c_{\mu}\right\|_{\sigma}}{q}\right),
$$

then for $\|u\|=\left(\frac{4 \lambda\left\|c_{\mu}\right\|_{\sigma}}{q}\right)^{1 /(2-q)}:=r(\mu, \lambda)$ one has

$$
J_{2}(u) \geq \delta(\mu, \lambda):=C\left[\lambda\left\|c_{\mu}\right\|_{\sigma}\right]^{2 /(2-q)} .
$$

Then, by $\left(H f_{c}\right)$, for suitably small $\mu>0$ one has $r(\mu, \lambda)<\rho(\lambda)$, so that

$$
J_{\mu, \lambda}(u) \geq \delta(\mu, \lambda)>0
$$

for $\|u\|=r(\mu, \lambda)$. Note that the claim of the lemma is true also for $\mu=0$ since in this case $c_{\mu} \equiv 0$ and then $J_{2}$ has a strict minimum at the origin.

By the same argument

$$
J_{1}^{ \pm}(u)=\frac{\lambda}{q} \int_{\Omega} d(x)\left|u^{ \pm}\right|^{q}-\frac{a}{2}\left\|u^{ \pm}\right\|_{2}^{2}-\int_{\Omega} G\left(x, u^{ \pm}\right) \geq 0
$$

for $\|u\| \leq \rho^{ \pm}(\lambda)$ and

$$
J_{2}^{ \pm}(u)=\frac{1}{2}\|u\|^{2}-\frac{\lambda}{q} \int_{\Omega} c_{\mu}(x)\left|u^{ \pm}\right|^{q} \geq \frac{1}{2}\|u\|^{2}-\frac{\lambda}{q} \int_{\Omega} c_{\mu}(x)|u|^{q},
$$

so one gets the same results for $J_{\mu, \lambda}^{ \pm}$.

The fact that the functionals are bounded from below in $B_{r}$ is trivial, by the hypotheses $\left(H f_{a}\right)$ and $\left(H g_{1}\right)$.

Lemma 3.4. Under the hypotheses of Theorem 2.1, for every $\lambda, \mu>0$, there exists $\phi \in H_{0}^{1}(\Omega)$ and $\bar{t}>0$ such that $J_{\mu, \lambda}(t \phi)<0$ for $t \in(0, \bar{t})$.

The same property holds for $J_{\mu, \lambda}^{ \pm}(u)$.

Proof. Once that $\lambda$ and $\mu$ are fixed, consider $\Omega_{\mu}$ as in $\left(H f_{d}\right)$ and a non-negative function $\phi \equiv 0$ with support in $\Omega_{\mu}$, then (using estimate (3.3))

$$
\begin{aligned}
J_{\mu, \lambda}(t \phi) & =\frac{t^{2}}{2}\left(\|\phi\|^{2}-a\|\phi\|_{2}^{2}\right)-\int_{\Omega} G(x, t \phi)-\frac{t^{q}}{q} \lambda \int_{\Omega} b_{\mu}(x) \phi^{q} \\
& \leq \frac{t^{2}}{2}\left(\|\phi\|^{2}-(a-\varepsilon)\|\phi\|_{2}^{2}\right)+\frac{t^{p}}{p} D_{\varepsilon}\|\phi\|_{p}^{p}-\frac{t^{q}}{q} \lambda \int_{\Omega} b_{\mu}(x) \phi^{q}
\end{aligned}
$$


where $\int_{\Omega} b_{\mu}(x) \phi^{q} \geq M_{\mu}\|\phi\|_{q}^{q}>0$ by $\left(H f_{d}\right)$; since the least power is $q$, one has $J_{\mu, \lambda}(t \phi)<0$ for small $t>0$.

The same holds for $J_{\mu, \lambda}^{+}(t \phi)$, and for $J_{\mu, \lambda}^{-}(-t \phi)$.

Now we may prove Theorem 2.1.

Proof of Theorem 2.1. For any $\lambda>0$ and $\mu \in(0, \bar{\mu}(\lambda))$, by Lemmas 3.3, 3.4 and the (PS) condition in Lemma $3.2, J_{\mu, \lambda}^{+}$has a strictly negative minimum in $B_{r}$, where $r$ is the radius obtained in Lemma 3.3; this is a first nontrivial non-negative solution.

Let now $u \geq 0$ be nontrivial, then

$$
J_{\mu, \lambda}^{+}(t u)=\frac{t^{2}}{2}\left(\|u\|^{2}-a\|u\|_{2}^{2}\right)-\int_{\Omega} G(x, t u)-\frac{t^{q}}{q} \lambda \int_{\Omega} b_{\mu}(x)|u|^{q}
$$

thus, we may use estimate (3.4) with $M$ so large that $\left(\|u\|^{2}-a\|u\|_{2}^{2}-M\|u\|_{2}^{2}\right)<0$ and then

$$
J_{\mu, \lambda}^{+}(t u) \leq \frac{t^{2}}{2}\left(\|u\|^{2}-a\|u\|_{2}^{2}-M\|u\|_{2}^{2}\right)+\int_{\Omega} E_{M}+\frac{t^{q}}{q} \lambda\left\|b_{\mu}\right\|_{\sigma}\|u\|^{q} \rightarrow-\infty
$$

for $t \rightarrow+\infty$; hence, one has another critical point, by Mountain Pass Theorem, whose level is at least the $\delta>0$ obtained in Lemma 3.3, which implies that it corresponds to a second nontrivial non-negative solution.

The same argument gives two nontrivial non-positive solutions as critical points of $J_{\mu, \lambda}^{-}$.

The two mountain pass levels may be characterized as

$$
c^{ \pm}=\inf _{\gamma \in \Gamma^{ \pm}} \sup _{t \in[0,1]} J_{\mu, \lambda}^{ \pm}(\gamma(t)),
$$

where

$$
\Gamma^{ \pm}=\left\{\gamma \in \mathcal{C}\left([0,1] ; H_{0}^{1}\right): \quad \gamma(0)=0, \quad \gamma(1)= \pm t_{0} \phi_{1}\right\}
$$

and $t_{0}>0$ is suitably large.

Finally, we prove the existence of a fifth nontrivial solution for $\lambda$ small enough, when $a \geq \lambda_{1}$ and $G \geq 0$.

Proof of Theorem 2.2. Let $a \in\left[\lambda_{k}, \lambda_{k+1}\right)$ for some $k \in \mathbb{N}$ and $H_{k}=\operatorname{span}\left\{\phi_{1}, . ., \phi_{k}\right\}$. The nontrivial solution will be obtained using a linking structure. Namely, we will obtain the critical level

$$
c_{e}=\inf _{\gamma \in \Gamma_{e}} \sup _{u \in \gamma\left(Q_{e}\right)} J_{\mu, \lambda}(u)
$$

where

$$
\begin{gathered}
\Gamma_{e}=\left\{\gamma \in \mathcal{C}\left(Q_{e}, H_{0}^{1}\right):\left.\gamma\right|_{\partial Q_{e}}=i d\right\}, \\
Q_{e}=\left\{u+t e: u \in H_{k}, \quad t \geq 0, \quad\|u+t e\| \leq R\right\},
\end{gathered}
$$

the function $e$ is any nonzero function in $H_{k}^{\perp}$ and $R$ a suitably large real.

We will need to prove the existence of constants $\eta, \widetilde{\eta}$ such that

$$
J_{\mu, \lambda}(u) \geq \eta>0 \text { in } S_{\rho} \cap H_{k}^{\perp}, \text { for a suitable } \rho \in(0, R),
$$




$$
J_{\mu, \lambda}(u) \leq \widetilde{\eta}<\eta \text { in } \partial Q_{e}, \text { the relative boundary of } Q_{e} ;
$$

actually $S_{\rho} \cap H_{k}^{\perp}$ links with $\partial Q_{e}$ and then the Linking Theorem states that $c_{e}$ is in fact a critical level for $J_{\mu, \lambda}$.

We may estimate $\left.\left|\int_{\Omega} b_{\mu}(x)\right| u\right|^{q} \mid \leq\left(\|d\|_{\sigma}+\left\|c_{\mu}\right\|_{\sigma}\right)\|u\|_{2^{*}}^{q}$; if we set $\mu \in[0, A)$, then by $\left(H f_{c}\right)$, we obtain $\left.\left|\int_{\Omega} b_{\mu}(x)\right| u\right|^{q} \mid \leq C_{A}\|u\|^{q}$ for a suitable constant $C_{A}$.

Let $u \in H_{k}^{\perp}$; we use estimate (3.3) with $\varepsilon<\lambda_{k+1}-a$ and obtain

$$
\begin{aligned}
J_{\mu, \lambda}(u) & \geq \frac{\|u\|^{2}-a\|u\|_{2}^{2}}{2}-\lambda \frac{C_{A}}{q}\|u\|^{q}-\frac{\varepsilon}{2}\|u\|_{2}^{2}-\frac{D_{\varepsilon}}{p}\|u\|_{p}^{p} \\
& \geq \frac{\lambda_{k+1}-a-\varepsilon}{2 \lambda_{k+1}}\|u\|^{2}-\lambda \frac{C_{A}}{q}\|u\|^{q}-D_{\varepsilon} \frac{C}{p}\|u\|^{p} ;
\end{aligned}
$$

since the coefficient of the quadratic term is positive, there exists a small enough $\bar{\lambda}>0$ such that for $\lambda \in[0, \bar{\lambda})$ the above function of $\|u\|$ is greater than a function with a positive maximum, that is, (3.12) above is satisfied for suitable $\rho, \eta>0$, and holds uniformly for $\lambda \in[0, \bar{\lambda})$ and $\mu \in[0, A)$.

Now let $u \in H_{k}$; then

$$
\begin{aligned}
J_{\mu, \lambda}(u) & \leq \frac{\lambda_{k}-a}{2}\|u\|_{2}^{2}+\frac{\lambda}{q} C_{A}\|u\|^{q}-\int_{\Omega} G(x, u) \\
& \leq \frac{\lambda}{q} C_{A}\|u\|^{q}-\frac{M}{2}\|u\|_{2}^{2}+E_{M}|\Omega|,
\end{aligned}
$$

where we used estimate (3.4) with any $M>0$. Since the norms are equivalent in $H_{k},(3.15)$ implies that the set $T=\left\{u \in H_{k}: J_{\mu, \lambda}(u) \geq 0\right.$ for some $\lambda \in[0, \bar{\lambda}]$ and $\left.\mu \in[0, A)\right\}$ is bounded, namely, $\|u\| \leq C_{T}$ in $T$; then from (3.14), using also the hypothesis that $G \geq 0$, we get $J_{\mu, \lambda}(u) \leq \frac{\lambda}{q} C_{A} C_{T}^{q} \rightarrow 0$ for $\lambda \rightarrow 0$. We conclude that, for suitably small $\lambda$ and $\mu \in[0, A)$,

$$
J_{\mu, \lambda} \leq \widetilde{\eta}<\eta \text { in } H_{k}
$$

Finally, given any nonzero $e \in H_{k}^{\perp}$, we estimate, using again (3.4) with $M$ so large that $\left(\|u\|^{2}-a\|u\|_{2}^{2}-M\|u\|_{2}^{2}\right)<0$ for every $u \in\left(H_{k} \oplus\{t e ; t \in \mathbb{R}\}\right)$, and we obtain

$$
J_{\mu, \lambda}(u) \leq \frac{\|u\|^{2}-a\|u\|_{2}^{2}}{2}+\frac{\lambda}{q} C_{A}\|u\|_{2^{*}}^{q}-M\|u\|_{2}^{2}+E_{M}|\Omega|
$$

then (since $\lambda$ is already bounded) we have $J_{\mu, \lambda} \leq 0$ in $S_{R} \cap\left(H_{k} \oplus\{t e ; t \in \mathbb{R}\}\right)$, for suitably large $R>0$ depending on $e$ (again the norms are equivalent). This estimate and (3.16) complete the proof of (3.13) and then we get that $c_{e}$ is in fact a critical level. Moreover, by (3.12), $c_{e} \geq \eta>0$, while the trivial and the other four solutions (when they exist) are at a lower level, actually, one may estimate the mountain pass levels in (3.8) by considering paths $\gamma \in \Gamma^{ \pm}$whose images are contained in $H_{k}$; by estimate (3.16) the maxima on such paths are at most $\widetilde{\eta}<\eta$, then $c^{ \pm}<\eta$ and this implies that $c_{e}$ corresponds to a different nontrivial solution. 


\section{The critical case}

In the critical case, even assuming the Ambrosetti-Rabinowitz condition $\left(\mathrm{Hg}_{2}\right)-(\mathrm{ii})$, the (PS) condition does not hold in general at every level of the functional. For this reason, in order to prove Theorems 2.4 and 2.5, we will verify that the infsup levels lie where (PS) holds.

We will need to consider two distinct problems. In order to prove Theorem 2.4 we have to estimate the mountain pass levels for any $\lambda>0$, while for Theorem 2.5 we will estimate the linking level, for suitably small $\lambda$.

Throughout this section we will consider Problem (2.1) with $N \geq 3$ and $p=2^{*}=\frac{2 N}{N-2}$.

We will denote by $S$ the best constant of the embedding of $H_{0}^{1}$ in $L^{p}$, namely

$$
S=\inf \left\{\frac{\|u\|^{2}}{\|u\|_{p}^{2}}: u \in H_{0}^{1}(\Omega) \backslash\{0\}\right\} .
$$

Remark 4.1. The nonlinearity in Problem (2.1) is odd with respect to the unknown $u$, which implies that for each non-negative nontrivial solution $u$, one has the non-positive nontrivial solution $-u$; then we will not consider $J_{\mu, \lambda}^{-}$any more and concentrate on the look for nonnegative solutions.

\subsection{The (PS) condition in the critical case}

First we analyze the (PS) levels of the functionals.

Lemma 4.2. Consider the functional

$$
J_{\mu, \lambda}(u)=\frac{1}{2}\left(\|u\|^{2}-a\|u\|_{2}^{2}\right)-\int_{\Omega} \lambda F_{\mu}(x, u)-\frac{1}{p}\|u\|_{p}^{p}
$$

with any $a \in \mathbb{R} ;$ if hypothesis $\left(H f_{a}\right)$ holds, then

(i) if $b_{\mu}(x) \leq 0$ the $(P S)_{c}$ condition holds for $c<\frac{1}{N} S^{N / 2}$;

(ii) for any $\varepsilon, A>0$, there exists $\lambda^{*}>0$ such that the $(P S)_{c}$ condition holds provided $c<$ $\frac{1}{N} S^{N / 2}-\varepsilon, \mu \in[0, A)$ and $\lambda \in\left[0, \lambda^{*}\right)$;

(iii) if there exists a minimal energy solution $u_{0}$, then the $(P S)_{c}$ condition holds for $c<$ $J_{\mu, \lambda}\left(u_{0}\right)+\frac{1}{N} S^{N / 2}$.

The same properties hold for $J_{\mu, \lambda}^{+}$.

Proof. Suppose $J_{\mu, \lambda}\left(u_{n}\right) \rightarrow c$ and $J_{\mu, \lambda}^{\prime}\left(u_{n}\right) \rightarrow 0$.

Since for any $\Theta \in(2, p)$, one has $\left(\frac{\Theta}{2}-1\right) a s^{2}+\left(\frac{\Theta}{p}-1\right) s^{p} \leq C$, we deduce as in Lemma 3.2 that the sequence $\left\{u_{n}\right\}$ is bounded. Then we may assume $u_{n} \rightarrow \bar{u}$ weakly in $H_{0}^{1}$, almost everywhere, and strongly in $L^{r}$ for $r<p$; moreover, $\left|u_{n}\right|^{p-2} u_{n} \rightarrow|\bar{u}|^{p-2} \bar{u}$ in $L^{p^{\prime}}$ (the dual of $L^{p}$ ) and then $\bar{u}$ is a solution of Problem (2.1). As a consequence we may write

$$
J_{\mu, \lambda}(\bar{u})=J_{\mu, \lambda}(\bar{u})-\frac{1}{2} J_{\mu, \lambda}^{\prime}(\bar{u})[\bar{u}]=\left(\frac{1}{2}-\frac{1}{p}\right)\|\bar{u}\|_{p}^{p}+\lambda\left(\frac{1}{2}-\frac{1}{q}\right) \int_{\Omega} b_{\mu}(x)|\bar{u}|^{q} .
$$


By Brézis-Lieb [15] we know that $\left\|u_{n}\right\|_{p}^{p}-\left\|u_{n}-\bar{u}\right\|_{p}^{p} \rightarrow\|\bar{u}\|_{p}^{p}$, while by weak convergence $\left\|u_{n}\right\|^{2}-$ $\left\|u_{n}-\bar{u}\right\|^{2} \rightarrow\|\bar{u}\|^{2}$, then

$$
\begin{gathered}
J_{\mu, \lambda}\left(u_{n}\right)=J_{\mu, \lambda}(\bar{u})+\frac{1}{2}\left\|u_{n}-\bar{u}\right\|^{2}-\frac{1}{p}\left\|u_{n}-\bar{u}\right\|_{p}^{p}+\sigma(1) \rightarrow c, \\
J_{\mu, \lambda}^{\prime}\left(u_{n}\right)\left[u_{n}\right]=J_{\mu, \lambda}^{\prime}(\bar{u})[\bar{u}]+\left\|u_{n}-\bar{u}\right\|^{2}-\left\|u_{n}-\bar{u}\right\|_{p}^{p}+\sigma(1) \rightarrow 0,
\end{gathered}
$$

where $\sigma(1)$ represents a quantity that goes to zero.

Since $J_{\mu, \lambda}^{\prime}(\bar{u})[\bar{u}]=0,(4.3)$ implies that (up to a subsequence)

$$
\left\|u_{n}-\bar{u}\right\|^{2} \rightarrow b, \quad\left\|u_{n}-\bar{u}\right\|_{p}^{p} \rightarrow b
$$

for some $b \geq 0$; if $b=0$ this means $u_{n} \rightarrow \bar{u}$ strongly. Otherwise, by (4.1) and (4.2)

$$
J_{\mu, \lambda}\left(u_{n}\right)=\left(\frac{1}{2}-\frac{1}{p}\right)\|\bar{u}\|_{p}^{p}+\lambda\left(\frac{1}{2}-\frac{1}{q}\right) \int_{\Omega} b_{\mu}(x)|\bar{u}|^{q}+\frac{1}{2}\left\|u_{n}-\bar{u}\right\|^{2}-\frac{1}{p}\left\|u_{n}-\bar{u}\right\|_{p}^{p}+\sigma(1) \rightarrow c
$$

and taking limit we get

$$
c=\frac{p-2}{2 p}\|\bar{u}\|_{p}^{p}+\lambda \frac{q-2}{2 q} \int_{\Omega} b_{\mu}(x)|\bar{u}|^{q}+\frac{p-2}{2 p} b .
$$

By Sobolev inequalities (remember that $\frac{p-2}{2 p}=\frac{1}{N}$ ) we have $\left\|u_{n}-\bar{u}\right\|^{2} \geq S\left\|u_{n}-\bar{u}\right\|_{p}^{2}$, then $b \geq S b^{2 / p}$, that is $b^{\frac{p-2}{p}}=b^{2 / N} \geq S$, and then

$$
c \geq \frac{1}{N}\left(S^{N / 2}+\|\bar{u}\|_{p}^{p}\right)+\lambda \frac{q-2}{2 q} \int_{\Omega} b_{\mu}(x)|\bar{u}|^{q} .
$$

If $b_{\mu}(x) \leq 0$, then this gives contradiction if $c<\frac{1}{N} S^{N / 2}$, so we have proved the claim (i).

Now we estimate (using, as in the proof of Theorem 2.2, that $\left.\left|\int_{\Omega} b_{\mu}(x)\right| u\right|^{q} \mid \leq C_{A}\|u\|_{p}^{q}$ for $\mu \in[0, A))$

$$
c \geq \frac{1}{N}\left(S^{N / 2}+\|\bar{u}\|_{p}^{p}\right)-\lambda C_{A} \frac{2-q}{2 q}\|\bar{u}\|_{p}^{q}
$$

since the minimum of $t^{p}-\lambda N C_{A} \frac{2-q}{2 q} t^{q}$ goes to zero as $\lambda \rightarrow 0^{+}$, one has that the (PS) condition holds below $\frac{1}{N} S^{N / 2}-\varepsilon$ for suitably small $\lambda$ (depending also on $A$ ); this proves the claim (ii).

Finally, let $u_{0}$ be a minimal energy solution; by identity (4.1) and inequality (4.4) we get

$$
c \geq J_{\mu, \lambda}(\bar{u})+\frac{1}{N} S^{N / 2} \geq J_{\mu, \lambda}\left(u_{0}\right)+\frac{1}{N} S^{N / 2},
$$

then, in the case of the claim (iii), we may guarantee (PS) $)_{c}$ below the level $J_{\mu, \lambda}\left(u_{0}\right)+\frac{1}{N} S^{N / 2}$.

For $J_{\mu, \lambda}^{+}\left(u_{n}\right)$ one proceeds in the same way: in fact $\left(u_{n}^{+}\right)^{p-1} \rightarrow\left(\bar{u}^{+}\right)^{p-1}$, then one may deduce that $\bar{u} \geq 0$ and apply Brézis-Lieb to the $\mathcal{C}^{1}$-function $t \mapsto\left(t^{+}\right)^{p}$. 


\subsection{Estimates of the infsup levels}

Now we need to estimate the infsup levels obtained in the proofs of Theorems 2.1 and 2.2; actually, the nonlinearity $g(x, u)=|u|^{2^{*}-2} u$ satisfies the conditions $\left(H g_{0} \ldots H g_{3}\right)$ with $p=2^{*}$ in $\left(H g_{1}\right)$, then all the estimates obtained for these theorems also hold in the critical case, except for the (PS) condition in Lemma 3.2.

As is classical in the literature, we will need to consider some compact support approximations of the instanton functions which realize the best Sobolev constant $S$ of the embedding $H_{1}\left(\mathbb{R}^{N}\right) \subset L^{p}\left(\mathbb{R}^{N}\right)$. Namely, consider

$$
\begin{gathered}
\Psi_{1}(x)=(N(N-2))^{(N-2) / 4} \frac{1}{\left(1+|x|^{2}\right)^{(N-2) / 2}}, \\
\Psi_{\nu}(x)=\nu^{(2-N) / 2} \Psi(x / \nu)=(N(N-2))^{(N-2) / 4}\left(\frac{\nu}{\left(\nu^{2}+|x|^{2}\right)}\right)^{(N-2) / 2},
\end{gathered}
$$

and consider a ball $B_{2 \xi} \subseteq \Omega$ and a function $\rho_{\xi} \in \mathcal{C}^{\infty}(\Omega ;[0,1])$ defined as 1 in $B_{\xi}$ and 0 in $\Omega \backslash \bar{B}_{2 \xi}$; then we set

$$
\psi_{\nu}(x)=\rho_{\xi}(x) \Psi_{\nu}(x) .
$$

With this definition one has the following estimates (see $[7,16]$ ), which will be used in the proofs of this section.

\section{Lemma 4.3.}

$$
\begin{aligned}
& \left\|\psi_{\nu}\right\|^{2}=S^{N / 2}+O\left(\nu^{N-2}\right), \\
& \left\|\psi_{\nu}\right\|_{p}^{p}=S^{N / 2}+O\left(\nu^{N}\right), \\
& \left\|\psi_{\nu}\right\|_{2}^{2}= \begin{cases}C \nu^{2}+O\left(\nu^{N-2}\right) & \text { for } N \geq 5, \\
C \nu^{2}|\ln (\mu)|+O\left(\nu^{2}\right) & \text { for } N=4, \\
O(\nu) & \text { for } N=3,\end{cases}
\end{aligned}
$$

where $O(f(\nu))$ represents a function of $\nu$ which is bounded in module by $C f(\nu)$ when $\nu \rightarrow 0$. Moreover, for some $C, C^{\prime}>0$ and $\nu$ small

$$
\begin{aligned}
& C^{\prime} \nu^{(N-2) / 2} \geq\left\|\psi_{\nu}\right\|_{p-1}^{p-1} \geq C \nu^{(N-2) / 2}, \\
& C^{\prime} \nu^{(N-2) q / 2} \geq\left\|\psi_{\nu}\right\|_{q}^{q} \quad \geq C \nu^{(N-2) q / 2}, \quad \text { provided } q<\frac{N}{N-2} .
\end{aligned}
$$

First, as a consequence of Lemma 4.2 , we obtain

Proposition 4.4. Under the hypotheses of Theorem 2.4, for $\lambda>0$ and $\mu \in(0, \bar{\mu}(\lambda))$, there exists a non-negative nontrivial solution $u_{0}$ of Problem (2.1), verifying that the corresponding critical level $J_{\mu, \lambda}^{+}\left(u_{0}\right)$ is negative and $\left\|u_{0}\right\|<r$, where $r$ is the radius obtained in Lemma 3.3.

Proof. Suppose for sake of contradiction that all critical points of $J_{\mu, \lambda}^{+}$are at non-negative level. Then, by point (iii) in Lemma 4.2, the (PS) condition would hold below $\frac{1}{N} S^{N / 2}$; as a consequence, arguing as in the first part of the proof of Theorem 2.1, we would get a critical point at negative level, by minimization in $B_{r}$. This proves the existence of a non-negative nontrivial solution $u_{0}$ at a negative level. 
In order to prove that $\left\|u_{0}\right\|<r$ consider

$$
j(t):=J_{\mu, \lambda}\left(t u_{0}\right)=\frac{t^{2}}{2}\left(\left\|u_{0}\right\|^{2}-a\left\|u_{0}\right\|_{2}^{2}\right)-\frac{t^{q}}{q} \lambda \int_{\Omega} b_{\mu}(x)\left|u_{0}\right|^{q}-\frac{t^{p}}{p}\left\|u_{0}\right\|_{p}^{p} ;
$$

we know that $t=1$ is a critical point at negative level for $j(t)$ and that if $\left\|\widetilde{t} u_{0}\right\|=r$ then $j(\widetilde{t}) \geq \delta>0$, by Lemma 3.3. This implies that, in the expression of $j$, the coefficient of $t^{q}$ is negative and that of $t^{2}$ is positive; as a consequence $j(t)<0$ for $t \in(0,1)$, and then $\widetilde{t}>1$, that is, $\left\|u_{0}\right\|<r$.

Then, we consider the mountain pass levels; in this case, we use the same infsup characterization (3.8), but we will need to take a different class of paths. Let then $\lambda>0$ and $\mu \in(0, \bar{\mu}(\lambda))$ as determined in Lemma 3.3; our candidate critical level is

$$
\begin{gathered}
c^{+}(\nu)=\inf _{\gamma \in \Gamma_{\nu}^{+}} \sup _{t \in[0,1]} J_{\mu, \lambda}^{+}(\gamma(t)), \\
\Gamma_{\nu}^{+}=\left\{\gamma \in \mathcal{C}\left([0,1] ; H_{0}^{1}\right): \quad \gamma(0)=u_{0}, \quad \gamma(1)=u_{0}+t_{0} \psi_{\nu}\right\},
\end{gathered}
$$

where $u_{0}$ is the non-negative solution of Proposition 4.4, $\psi_{\nu}$ is taken with support in $\Omega_{\mu}$ from condition $\left(H f_{d}\right)$ and $t_{0}$ (depending on $\nu$ ) is chosen such that $\left\|u_{0}+t_{0} \psi_{\nu}\right\|>r$ and $J_{\mu, \lambda}^{+}\left(u_{0}+\right.$ $\left.t_{0} \psi_{\nu}\right)<0$; the fact that this is possible is a consequence of inequality (4.18) in the proof of the next lemma.

Lemma 4.5. Under the hypotheses of Theorem 2.4, there exists $\nu>0$ such that

$$
c^{+}(\nu)<J_{\mu, \lambda}\left(u_{0}\right)+\frac{1}{N} S^{N / 2} .
$$

Proof. We need to evaluate, for $t \geq 0$,

$$
\begin{aligned}
J_{\mu, \lambda}\left(u_{0}+t \psi_{\nu}\right)= & \frac{1}{2}\left(\left\|u_{0}\right\|^{2}-a\left\|u_{0}\right\|_{2}^{2}\right)+\frac{t^{2}}{2}\left(\left\|\psi_{\nu}\right\|^{2}-a\left\|\psi_{\nu}\right\|_{2}^{2}\right)+ \\
& +t \int_{\Omega}\left(\nabla u_{0} \nabla \psi_{\nu}-a u_{0} \psi_{\nu}\right)-\frac{\lambda}{q} \int_{\Omega} b_{\mu}(x)\left|u_{0}+t \psi_{\nu}\right|^{q}-\frac{1}{p}\left\|u_{0}+t \psi_{\nu}\right\|_{p}^{p} ;
\end{aligned}
$$

we will use the following estimates, deduced from the inequalities $(1+s)^{p} \geq 1+s^{p}+s p$ and $(1+s)^{q} \geq 1+s q$ for any $s \geq 0$ :

$$
\begin{gathered}
\left\|u_{0}+t \psi_{\nu}\right\|_{p}^{p} \geq\left\|u_{0}\right\|_{p}^{p}+t^{p}\left\|\psi_{\nu}\right\|_{p}^{p}+t p \int_{\Omega} u_{0}^{p-1} \psi_{\nu}, \\
\int_{\Omega} b_{\mu}(x)\left|u_{0}+t \psi_{\nu}\right|^{q} \geq \int_{b_{\mu} \geq 0} b_{\mu}(x)\left(u_{0}^{q}+q u_{0}^{q-1} t \psi_{\nu}\right)+\int_{b_{\mu}<0} b_{\mu}(x) u_{0}^{q},
\end{gathered}
$$

where we used the fact that $\psi_{\nu}=0$ when $b_{\mu}(x)<0$. Moreover, since $u_{0}$ is a solution,

$$
t \int_{\Omega}\left(\nabla u_{0} \nabla \psi_{\nu}-a u_{0} \psi_{\nu}\right)=\lambda \int_{\left(b_{\mu} \geq 0\right)} b_{\mu}(x) u_{0}^{q-1} t \psi_{\nu}+\int_{\Omega} u_{0}^{p-1} t \psi_{\nu}
$$


and these two terms annihilate with the corresponding ones in (4.15) and (4.16), so that Eq. (4.14) reads

$$
J_{\mu, \lambda}\left(u_{0}+t \psi_{\nu}\right) \leq J_{\mu, \lambda}\left(u_{0}\right)+\frac{t^{2}}{2}\left(\left\|\psi_{\nu}\right\|^{2}-a\left\|\psi_{\nu}\right\|_{2}^{2}\right)-\frac{t^{p}}{p}\left\|\psi_{\nu}\right\|_{p}^{p} .
$$

For $N \geq 4$, the claim follows as in [7] since $a>0$.

Observe that (4.18) also implies that it is possible to find $t_{0}(\nu)$ as required for the definition in (4.13).

Proof of the case $N=3$. We consider now the case $N=3$, which implies $p=6$.

Observe that if $\Omega_{\mu}$ is as in $\left(H f_{d}\right)$ then, in $\Omega_{\mu},-\Delta u_{0} \geq 0$ and then either $u_{0} \equiv 0$ or $u_{0}>0$.

In the second case, passing if necessary to a ball contained in $\Omega_{\mu}$, we have $u_{0} \geq C>0$, then we use in place of (4.15) the estimate

$$
\left\|u_{0}+t \psi_{\nu}\right\|_{6}^{6} \geq\left\|u_{0}\right\|_{6}^{6}+t^{6}\left\|\psi_{\nu}\right\|_{6}^{6}+6 t \int_{\Omega} \psi_{\nu} u_{0}^{5}+6 t^{5} \int_{\Omega} \psi_{\nu}^{5} u_{0},
$$

so that (4.14) becomes (using $b_{\mu}(x)>M_{\mu}$ and $u_{0} \geq C>0$ )

$$
J_{\mu, \lambda}\left(u_{0}+t \psi_{\nu}\right) \leq J_{\mu, \lambda}\left(u_{0}\right)+\frac{t^{2}}{2}\left(\left\|\psi_{\nu}\right\|^{2}-a\left\|\psi_{\nu}\right\|_{2}^{2}\right)-\frac{t^{6}}{6}\left\|\psi_{\nu}\right\|_{6}^{6}-C t^{5}\left\|\psi_{\nu}\right\|_{5}^{5} .
$$

The maximum of the above function of $t \geq 0$ is reached for some $t_{\nu}$, which is bounded and bounded away from zero with respect to $\nu$ when this is small, actually, by (4.7), (4.8), (4.9) and (4.10), when $\nu \rightarrow 0$ the right hand side of (4.20) converges uniformly on compact sets to $\frac{t^{2}}{2} S^{3 / 2}-\frac{t^{6}}{6} S^{3 / 2}$, which has a unique maximum and then goes to $-\infty$. Then we deduce, using the estimates of Lemma 4.3

$$
\begin{aligned}
J_{\mu, \lambda}\left(u_{0}+t \psi_{\nu}\right) & \leq J_{\mu, \lambda}\left(u_{0}\right)+\frac{t_{\nu}^{2}}{2}\left(\left\|\psi_{\nu}\right\|^{2}-a\left\|\psi_{\nu}\right\|_{2}^{2}\right)-\frac{t_{\nu}^{6}}{6}\left\|\psi_{\nu}\right\|_{6}^{6}-C t_{\nu}^{5}\left\|\psi_{\nu}\right\|_{5}^{5} \\
& \leq J_{\mu, \lambda}\left(u_{0}\right)+\frac{t_{\nu}^{2}}{2}\left(S^{3 / 2}+O(\nu)\right)-\frac{t_{\nu}^{6}}{6}\left(S^{3 / 2}+O\left(\nu^{3}\right)\right)-C t_{\nu}^{5} \sqrt{\nu} \\
& \leq J_{\mu, \lambda}\left(u_{0}\right)+\frac{t_{\nu}^{2}}{2} S^{3 / 2}-\frac{t_{\nu}^{6}}{6} S^{3 / 2}-C \sqrt{\nu}+O(\nu) .
\end{aligned}
$$

Since the maximum of the function $\frac{t^{2}}{2} S^{3 / 2}-\frac{t^{6}}{6} S^{3 / 2}$ is $\frac{1}{3} S^{3 / 2}$, for suitably small $\nu$, the claim is proved by virtue of the least order term $-C \sqrt{\nu}$.

Finally, we consider the case $u_{0} \equiv 0$ in $\Omega_{\mu}$; in this case the supports of $u_{0}$ and $\psi_{\nu}$ are disjoint, then

$$
J_{\mu, \lambda}\left(u_{0}+t \psi_{\nu}\right)=J_{\mu, \lambda}\left(u_{0}\right)+J_{\mu, \lambda}\left(t \psi_{\nu}\right)
$$

and we may consider

$$
J_{\mu, \lambda}\left(t \psi_{\nu}\right)=\frac{t^{2}}{2}\left(\left\|\psi_{\nu}\right\|^{2}-a\left\|\psi_{\nu}\right\|_{2}^{2}\right)-t^{6} \frac{1}{6}\left\|\psi_{\nu}\right\|_{6}^{6}-t^{q} \frac{\lambda}{q} \int_{\Omega} b_{\mu}(x) \psi_{\nu}^{q} ;
$$

using $\left(H f_{d}\right)$ and (4.11) we get $\int_{\Omega} b_{\mu}(x) \psi_{\nu}^{q} \geq M_{\mu}\|\psi\|_{q}^{q} \geq C \nu^{q / 2}$ and then, proceeding as we did with (4.20), we get

$$
J_{\mu, \lambda}\left(t \psi_{\nu}\right) \leq \frac{1}{3} S^{3 / 2}-C^{\prime} \nu^{q / 2}+O(\nu)
$$

since $q<2$, the least order term is $-C^{\prime} \nu^{q / 2}$ and then for suitably small $\nu>0$ we obtain the claim. 
With this result we may give the proof of Theorem 2.4

Proof of Theorem 2.4. Proposition 4.4 gives a first non-negative solution $u_{0}$, at a negative level. By point (iii) in Lemma 4.2, if the origin and $u_{0}$ were the unique critical points of $J_{\mu, \lambda}^{+}$, then $(\mathrm{PS})_{c}$ would hold below $J_{\mu, \lambda}^{+}\left(u_{0}\right)+\frac{1}{N} S^{N / 2}$; but in this case, for the $\nu$ given in Lemma 4.5 , the mountain pass level (4.12) would be a further critical point. This proves the existence of a second non-negative nontrivial solution. The non-positive solutions come from remark 4.1.

Now we consider the linking level obtained in Theorem 2.2.

Lemma 4.6. Under the hypotheses of Theorem 2.5, for any $A>0$, there exist $\nu, \varepsilon, \widetilde{\lambda}>0$ such that if $e_{\nu}$ is the component of $\psi_{\nu}$ orthogonal to $H_{k}$ then the infsup level $c_{e_{\nu}}$ in (3.9) satisfies

$$
c_{e_{\nu}}<\frac{1}{N} S^{N / 2}-\varepsilon
$$

for any $\lambda \in[0, \widetilde{\lambda})$ and $\mu \in[0, A)$.

Proof. Let $e_{\nu}$ be the component orthogonal to $H_{k}$ of the function $\psi_{\nu}$, and let $Y_{\nu}=H_{k} \oplus$ $\left\{t e_{\nu}: t \in \mathbb{R}\right\}$. For any given $u \in Y_{\nu}$ we have

$$
J_{\mu, \lambda}(t u)=\frac{t^{2}}{2}\left(\|u\|^{2}-a\|u\|_{2}^{2}\right)-\lambda \frac{t^{q}}{q} \int_{\Omega} b_{\mu}(x)|u|^{q}-\frac{t^{p}}{p}\|u\|_{p}^{p}
$$

we estimate, $\frac{s^{q}}{q} \leq 1+\frac{s^{2}}{2} C_{1}$ and we use again $\left.\left|\int_{\Omega} b_{\mu}(x)\right| u\right|^{q} \mid \leq C_{A}\|u\|_{p}^{q}$ for any $\mu \in[0, A)$, obtaining

$$
\left.\left|\frac{\lambda}{q} t^{q} \int_{\Omega} b_{\mu}(x)\right| u\right|^{q} \mid \leq \lambda C_{A}\left(1+C_{1} \frac{t^{2}}{2}\|u\|_{p}^{2}\right) .
$$

With this estimate, (4.25) implies

$$
J_{\mu, \lambda}(t u) \leq \lambda C_{A}+\frac{t^{2}}{2}\left[\|u\|^{2}-a\|u\|_{2}^{2}+\lambda C_{A} C_{1}\|u\|_{p}^{2}\right]-\frac{t^{p}}{p}\|u\|_{p}^{p}
$$

since the maximum of $A \frac{t^{2}}{2}-B \frac{t^{p}}{p}$ is for $t=\left(\frac{A}{B}\right)^{\frac{1}{p-2}}$ at level $\left(\frac{1}{2}-\frac{1}{p}\right) \frac{A^{N / 2}}{B^{N / 2-1}}$, we get

$$
\begin{aligned}
J_{\mu, \lambda}(t u) & \leq \lambda C_{A}+\frac{1}{N} \frac{\left[\|u\|^{2}-a\|u\|_{2}^{2}+\lambda C_{A} C_{1}\|u\|_{p}^{2}\right]^{N / 2}}{\left(\|u\|_{p}^{p}\right)^{N / 2-1}} \\
& \leq \lambda C_{A}+\frac{1}{N}\left[\frac{\|u\|^{2}-a\|u\|_{2}^{2}}{\|u\|_{p}^{2}}+\lambda C_{A} C_{1}\right]^{N / 2} .
\end{aligned}
$$

Now we consider

$$
m_{\nu}=\max \left\{\|u\|^{2}-a\|u\|_{2}^{2}: \quad u \in Y_{\nu},\|u\|_{p}=1\right\} .
$$

The maximum is assumed since $Y_{\nu}$ is finite dimensional, then let $u$ be a maximizer and write $u=\widetilde{y}+\tau e_{\nu}=y+\tau \psi_{\nu}$ with $y, \widetilde{y} \in H_{k}$. Observe that by triangular inequality and the estimates $(4.9)$

$$
\|y\|_{2} \leq\|u\|_{2}+\left\|\tau \psi_{\nu}\right\|_{2} \leq C\|u\|_{p}+|\tau|\left\|\psi_{\nu}\right\|_{2}=C+|\tau| O(\nu) ;
$$


using the inequality $|1+s|^{p} \geq 1+p s$, for any $s \in \mathbb{R}$, one gets

$$
1=\|u\|_{p}^{p}=\left\|y+\tau \psi_{\nu}\right\|_{p}^{p} \geq\left\|\tau \psi_{\nu}\right\|_{p}^{p}+p \int_{\Omega}\left(\tau \psi_{\nu}\right)^{p-1} y \geq|\tau|^{p}\left\|\psi_{\nu}\right\|_{p}^{p}-|\tau|^{p-1} C\left\|\psi_{\nu}\right\|_{p-1}^{p-1}\|y\|_{\infty}
$$

and using (4.29) ( $H_{k}$ is finite dimensional so the norms are equivalent for $y$ ), (4.8) and (4.10), this becomes

$$
1 \geq|\tau|^{p-1}\left[|\tau|\left(S^{N / 2}+\sigma(1)\right)-C \sigma(1)-|\tau| \sigma(1)\right] ;
$$

we conclude that $|\tau|$ (and then also $y$ by (4.29)) is bounded for $\nu \rightarrow 0$.

Now, from (4.30) and using the boundedness of $\tau$ we deduce

$$
1 \geq\left\|\tau \psi_{\nu}\right\|_{p}^{p}-C\left\|\psi_{\nu}\right\|_{p-1}^{p-1}\|y\|_{\infty} ;
$$

then we compute

$$
\begin{aligned}
m_{\nu} & =\left(y+\tau \psi_{\nu}, y+\tau \psi_{\nu}\right)-a\left(y+\tau \psi_{\nu}, y+\tau \psi_{\nu}\right)_{2} \\
& \leq\|y\|^{2}-a\|y\|_{2}^{2}+\tau^{2}\left(\left\|\psi_{\nu}\right\|^{2}-a\left\|\psi_{\nu}\right\|_{2}^{2}\right)+2|\tau|\left(\left|\left(y, \psi_{\nu}\right)\right|+a\left|\left(y, \psi_{\nu}\right)_{2}\right|\right) \\
& \leq\left(\lambda_{k}-a\right)\|y\|_{2}^{2}+\tau^{2}\left\|\psi_{\nu}\right\|_{p}^{2} \frac{\left\|\psi_{\nu}\right\|^{2}-a\left\|\psi_{\nu}\right\|_{2}^{2}}{\left\|\psi_{\nu}\right\|_{p}^{2}}+C\|y\|_{\infty}\left\|\psi_{\nu}\right\|_{1}
\end{aligned}
$$

where we exploited the regularity of $y$ to estimate $\left|\left(y, \psi_{\nu}\right)\right|=\left|\left(\Delta y, \psi_{\nu}\right)_{2}\right| \leq C\|y\|_{\infty}\left\|\psi_{\nu}\right\|_{1}$. By (4.31), and estimate (4.10)

$$
\tau^{2}\left\|\psi_{\nu}\right\|_{p}^{2} \leq\left(1+C\left\|\psi_{\nu}\right\|_{p-1}^{p-1}\|y\|_{\infty}\right)^{2 / p}=\left(1+O\left(\nu^{(N-2) / 2}\right)\|y\|_{\infty}\right) .
$$

By estimates (4.7), (4.8) and (4.9) (in the case $N \geq 5$ )

$$
\frac{\left\|\psi_{\nu}\right\|^{2}-a\left\|\psi_{\nu}\right\|_{2}^{2}}{\left\|\psi_{\nu}\right\|_{p}^{2}}=\frac{S^{N / 2}-a C \nu^{2}+O\left(\nu^{N-2}\right)}{\left(S^{N / 2}+O\left(\nu^{N}\right)\right)^{2 / p}}=S-a C \nu^{2}+O\left(\nu^{N-2}\right) .
$$

Using $\left\|\psi_{\nu}\right\|_{1}=O\left(\nu^{(N-2) / 2}\right)$, the equivalence of the norms of $y$, Eqs. (4.33) and (4.34) in (4.32) we obtain

$$
m_{\nu} \leq\left(\lambda_{k}-a\right)\|y\|^{2}+\left(S-a C \nu^{2}+O\left(\nu^{N-2}\right)+O\left(\nu^{(N-2) / 2}\right)\|y\|\right) .
$$

Since $\lambda_{k}<a$, we have two possibilities:

$$
\text { either } \quad\left(\lambda_{k}-a\right)\|y\|^{2}+O\left(\nu^{(N-2) / 2}\right)\|y\| \leq 0, \quad \text { or }
$$

$\left(a-\lambda_{k}\right)\|y\| \leq O\left(\nu^{(N-2) / 2}\right)$, which implies

$$
\|y\| O\left(\nu^{(N-2) / 2}\right)=O\left(\nu^{N-2}\right) ;
$$

in both cases, we may conclude from (4.35) that

$$
m_{\nu} \leq S-a C \nu^{2}+O\left(\nu^{N-2}\right)
$$

and then from (4.27)

$$
J_{\mu, \lambda}(t u) \leq \lambda C_{A}+\frac{1}{N}\left[S-a C \nu^{2}+O\left(\nu^{N-2}\right)+\lambda C_{A} C_{1}\right]^{N / 2} .
$$


To conclude, we have to fix suitable values for $\varepsilon, \nu$ and $\widetilde{\lambda}$ in order to satisfy the claim; first we may set $\nu, \varepsilon$ such that the term $\frac{1}{N}\left[S-a C \nu^{2}+O\left(\nu^{N-2}\right)\right]^{N / 2}$ in (4.39) is smaller than $\frac{1}{N} S^{N-2}-2 \varepsilon$, then for suitably small $\tilde{\lambda}$ the whole right hand side in (4.39) is smaller than $\frac{1}{N} S^{N / 2}-\varepsilon$, for any $\mu \in[0, A)$ and $\lambda \in[0, \widetilde{\lambda})$.

The case $N=4$ is similar: the unique difference is in Eqs. (4.34) and (4.38), where one uses the second of the estimates in (4.9) and then obtains $m_{\nu} \leq S-a C \nu^{2}|\ln (\nu)|+O\left(\nu^{2}\right)$.

At this point we may give the proof of Theorem 2.5:

Proof of Theorem 2.5. Given the value $A>0$, let $\nu, \varepsilon, \widetilde{\lambda}$ be as in Lemma 4.6, then point (ii) in Lemma 4.2 implies that for suitably small $\lambda$ the level $c_{e_{\nu}}$ is in fact critical, and then it corresponds to two nontrivial solutions, since the functional is even.

Since $a>\lambda_{1}$, we do not need to use the mountain pass characterization in (4.12) with Lemma 4.5 in order to estimate the level of the mountain pass solution; in fact, the same argument used in Theorem 2.5 shows that the mountain pass level as defined in (3.8) is lower than $c_{e_{\nu}}$ and then it lies at a level where (PS) holds.

Finally, we analyze what happens in the case $\mu=0$, that is, $b_{\mu}<-\delta_{d}<0$.

Proof of Theorem 2.6. Since $\mu=0$ the claim in Lemma 3.4 does not hold, so we do not have the minimum solutions.

However, by point (i) in Lemma 4.2 the $(\mathrm{PS})_{c}$ condition is satisfied below $\frac{1}{N} S^{N / 2}$, moreover, Lemma 3.3 holds and the whole proof of Theorem 2.2 works in the same way, then we may apply the Mountain Pass and the Linking Theorem, provided the corresponding levels are below $\frac{1}{N} S^{N / 2}$.

Finally, also Lemma 4.6 holds and again, since the mountain pass level is below the linking level, we conclude that for suitably small $\lambda>0$ both kinds of solution exist.

\section{The case $\left(f_{2}\right)$}

We sketch here the differences in the proofs which arise when considering the case $\left(f_{2}\right)$ instead of the case $\left(f_{1}\right)$.

Observe that most of the arguments just exploit the fact that $1<q<2$ and the estimate $\left.\left|\int_{\Omega} b_{\mu}(x)\right| u\right|^{q} \mid \leq C_{A}\|u\|^{q}$, which may be replaced with

$$
-C_{A}\|u\|^{q_{2}} \leq \int_{\Omega} \bar{c}_{\mu}(x)|u|^{q_{1}}-\bar{d}(x)|u|^{q_{2}} \leq C_{A}\|u\|^{q_{1}}
$$

these arguments are then extended straightforward to case $\left(f_{2}\right)$.

The real difference arises in Lemma 3.4: in this case the result will be given by the fact that the exponents are different, and not by the unboundedness of $b_{\mu}$, which is not assumed in hypothesis $\left(H f_{d}^{\prime}\right)$. Following the lines of the original proof, one can choose $\Omega_{\mu}$ as in $\left(H f_{d}^{\prime}\right)$ and again $\phi \equiv \equiv$ a non-negative function with support in $\Omega_{\mu}$, then

$$
J_{\mu, \lambda}(t \phi) \leq \frac{t^{2}}{2}\left(\|\phi\|^{2}-(a-\varepsilon)\|\phi\|_{2}^{2}\right)+\frac{t^{p}}{p} D_{\varepsilon}\|\phi\|_{p}^{p}-\frac{t^{q_{1}}}{q_{1}} \lambda \int_{\Omega} \bar{c}_{\mu}(x) \phi^{q_{1}}+\frac{t^{q_{2}}}{q_{2}} \lambda \int_{\Omega} \bar{d}(x) \phi^{q_{2}}
$$


where $\int_{\Omega} \bar{c}_{\mu}(x) \phi^{q_{1}} \geq M_{\mu}\|\phi\|_{q_{1}}^{q_{1}}>0$ and now the least power is $q_{1}$, then again $J_{\mu, \lambda}(t \phi)<0$ for small $t>0$.

In the proof of Proposition 4.4, when proving that $\left\|u_{0}\right\|<r$, one has now to deal with four different powers in the expression of $j(t)$; however in this case the unique unknown sign is that of the term in $t^{2}$, so that one may still prove that $j$ may have at most a minimum and then a maximum.

Some technical differences arise in the proof of Lemma 4.5; first, we get in place of (4.16)

$$
\int_{\Omega} \bar{c}_{\mu}(x)\left|u_{0}+t \psi_{\nu}\right|^{q_{1}}-\bar{d}(x)\left|u_{0}+t \psi_{\nu}\right|^{q_{2}} \geq \int_{\Omega} \bar{c}_{\mu}(x)\left(u_{0}^{q_{1}}+q_{1} u_{0}^{q_{1}-1} t \psi_{\nu}\right)-\int_{\Omega} \bar{d}(x) u_{0}^{q_{2}}
$$

and in place of $(4.17)$

$$
t \int_{\Omega}\left(\nabla u_{0} \nabla \psi_{\nu}-a u_{0} \psi_{\nu}\right)=\lambda \int_{\Omega}\left(\bar{c}_{\mu}(x) u_{0}^{q_{1}-1}-\bar{d}(x) u_{0}^{q_{2}-1}\right) t \psi_{\nu}+\int_{\Omega} u_{0}^{p-1} t \psi_{\nu}
$$

then we get again (4.18) since the term $\left(-\int_{\Omega} \bar{d}(x) u_{0}^{q_{2}-1} t \psi_{\nu}\right)$ which is not canceled between the above expressions is non-positive, and we may proceed as before.

Later in the same proof, the last term in (4.24) becomes

$$
t^{q} \frac{\lambda}{q}\left(-\int_{\Omega} \bar{c}_{\mu}(x) \psi_{\nu}^{q_{1}}+\int_{\Omega} \bar{d}(x) \psi_{\nu}^{q_{2}}\right) \leq-C^{\prime} \nu^{q_{1} / 2}+C^{\prime} \nu^{q_{2} / 2}
$$

and since $q_{1}<q_{2}$ we may conclude as before.

Observe that this last estimate is the unique place where we need the hypothesis that $d(x)$ is bounded in $\Omega_{\mu}$ (part of $\left(H f_{d}^{\prime}\right)$ ); in fact, this hypothesis could be removed except for the critical case in dimension $N=3$.

\section{Acknowledgment.}

This work was done while the first author was visiting Universidad de Santiago de Chile, with a postdoctoral position.

\section{References}

[1] A. Ambrosetti, H. Brezis, G. Cerami, Combined effects of concave and convex nonlinearities in some elliptic problems, J. Funct. Anal. 122 (2) (1994) 519-543.

[2] F. O. de Paiva, E. Massa, Multiple solutions for some elliptic equations with a nonlinearity concave at the origin, Nonlinear Anal. 66 (12) (2007) 2940-2946.

[3] D. G. De Figueiredo, J. P. Gossez, P. Ubilla, Local superlinearity and sublinearity for indefinite semilinear elliptic problems, J. Funct. Anal. 199 (2) (2003) 452-467.

[4] D. G. de Figueiredo, J.-P. Gossez, P. Ubilla, Multiplicity results for a family of semilinear elliptic problems under local superlinearity and sublinearity, J. Eur. Math. Soc. (JEMS) 8 (2) (2006) 269-286.

[5] K. Perera, Critical groups of pairs of critical points produced by linking subsets, J. Differential Equations 140 (1) (1997) 142-160. 
[6] V. Moroz, On the Morse critical groups for indefinite sublinear elliptic problems, Nonlinear Anal. 52 (5) (2003) 1441-1453.

[7] H. Brézis, L. Nirenberg, Positive solutions of nonlinear elliptic equations involving critical Sobolev exponents, Comm. Pure Appl. Math. 36 (4) (1983) 437-477.

[8] A. Capozzi, D. Fortunato, G. Palmieri, An existence result for nonlinear elliptic problems involving critical Sobolev exponent, Ann. Inst. H. Poincaré Anal. Non Linéaire 2 (6) (1985) $463-470$.

[9] M. Willem, Minimax theorems, Vol. 24 of Progress in Nonlinear Differential Equations and their Applications, Birkhäuser Boston Inc., Boston, MA, 1996.

[10] K. Perera, Multiplicity results for some elliptic problems with concave nonlinearities, J. Differential Equations 140 (1) (1997) 133-141.

[11] S. Wu, H. Yang, A class of resonant elliptic problems with sublinear nonlinearity at origin and at infinity, Nonlinear Anal. 45 (7, Ser. A: Theory Methods) (2001) 925-935.

[12] S. Li, S. Wu, H. Zhou, Solutions to semilinear elliptic problems with combined nonlinearities, J. Differential Equations 185 (1) (2002) 200-224.

[13] J. Sánchez, P. Ubilla, Uniqueness results for the one-dimensional $m$-Laplacian considering superlinear nonlinearities, Nonlinear Anal. 54 (5) (2003) 927-938.

[14] H. Brezis, L. Nirenberg, $H^{1}$ versus $C^{1}$ local minimizers, C. R. Acad. Sci. Paris Sér. I Math. 317 (5) (1993) 465-472.

[15] H. Brézis, E. Lieb, A relation between pointwise convergence of functions and convergence of functionals, Proc. Amer. Math. Soc. 88 (3) (1983) 486-490.

[16] N. Ghoussoub, Duality and perturbation methods in critical point theory, Vol. 107 of Cambridge Tracts in Mathematics, Cambridge University Press, Cambridge, 1993, with appendices by David Robinson. 